Background FHHNC is a rare autosomal recessive tubulopathy of the thick ascending limb due to inactivating mutations in the Claudin-16 and Claudin-19 genes which are responsible for the paracellular reabsorption of calcium and magnesium. Clinically, FHHNC is characterized by urinary tract infections, nephrolithiasis, nephrocalcinosis and progressive renal failure.

Objective To present clinical and molecular data on 2 siblings with FHHNC and early onset SND, found to have a new mutation in the Claudin-16 gene.

Methods A 16-year-old male and his 14-year-old sister were diagnosed with chronic kidney disease since early infancy, manifested by recurrent UTIs, polyuria and polydipsia, poor growth, mild mental retardation and delayed speech. SND was diagnosed at the age of 6 and 7 years. Both have hypomagnesemia, hypermagnesuria, hypercalciuria and nephrocalcinosis. Genotyping was performed by PCR using tetranucleotide repeat polymorphisms. Specific primer pairs of genomic DNA were used as template for sequencing the Cldin16 gene.

Results Exons 1, 2, 4 and 5 were amplified and revealed wild type sequencing but exon 3 failed in amplification by PCR. Long range PCR spanning exon 2 to 4 of the gene yielded a $2.5 \mathrm{~kb}$ fragment shared by the patients. Sequencing of this fragment reveal a $2630 \mathrm{bp}$ deletion including the entire exon 3 resulting in deletion and frame shift of Cldn16 protein.

Conclusion This family demonstrates the first identified homozygous mutation in the Cldn16 gene causing the deletion of an entire exon. This, however, is unlikely to explain the SND, since this gene is not expressed in the cochlea.

\section{PROTEOLYTICAL ENZYMES IN GLOMERULONEPHRITIS}

doi:10.1136/archdischild-2012-302724.1202

${ }^{1}$ A Ciuntu, ' $N$ Revenco, ${ }^{2}$ S Benesh. 'Pediatrics, USMF 'Nicolae Testemitanu'; '2Pediatrics, State Medical and Pharmaceutical University 'N. Testemitanu', Chisinau, Moldova

Aim of the investigation: Study of activity of proteolytic enzymes in children with acute (AGN) and chronic (CGN) glomerylonepritis.

Materials and Methods Were investigated 50 children with acute and chronic glomerulonephritis, age from 7 to 15 years, including 30 children with AGN plus nephrotic syndrome, and 20 children with CGN, nephrotic form, period of activation and remission. Control group including 20 practically healthy children. In serum determined activity of trypsin, a-antitrypsine, and $\mathrm{a}_{2}$-macroglobuline.

Results In AGN with nephrotic syndrome, at the initial stage of the process, as well as in activation of CGN, were determined significant increasing of trypsine, a-antitrypsine, and $\mathrm{a}_{2}$-macroglobuline in comparation to control group $(\mathrm{P}<0.01)$. The activity of $\mathrm{a}_{2}$-macroglobuline in children with AGN plus nephrotic syndrome ant the initial stage of disease was $11.9 \pm 1.42 \mathrm{~g} / \mathrm{l}, \mathrm{P}<0.001$, what 1.9 fold higher than in control group $(6.2 \pm 0.030 \mathrm{~g} / \mathrm{l})$. In activation of CGN, nephrotic form, the activity of $\mathrm{a}_{2}$-macroglobuline was in 1.5 fold higher the indexes of control group $(9.5 \pm 0.90 \mathrm{~g} / \mathrm{l}, \mathrm{P}<0.01)$.

In remission, the values of trypsin, a-antitrypsine, and $\mathrm{a}_{2}$-macroglobuline decreasing, but did not attain the basic level of control group, which suggests the persistence of activity of pathological process in kidneys.

Conclusion Determining of activity of proteolytic enzymes in serum in diagnostic criterion for determining of severity, activity of pathological process in kidneys, and determining the outcome the complications of glomerulonephritis.

\section{THE DIABETIC PREGNANCY AND OFFSPRING BLOOD PRESSURE IN CHILDHOOD: A SYSTEMATIC REVIEW AND META-ANALYSIS}

doi:10.1136/archdischild-2012-302724.1203
1.2A Aceti, 'S Santhakumaran, 'KM Logan, 'LH Philipps, 'E Prior, 'C Gale, 'MJ Hyde, 'N Modi. 'Academic Neonatal Medicine, Imperial College, London, UK; ${ }^{2}$ Neonatal Medicine, Alma Mater Studiorum, Bologna, Italy

Background and Aims Offspring of diabetic mothers (ODM) are at increased risk of the metabolic syndrome in later life. We aimed to perform a systematic review and meta-analysis of studies examining offspring systolic and diastolic blood pressure (SBP, DBP) in childhood in relation to maternal diabetes.

Methods Citations were identified in PubMed. Authors were contacted for additional data where necessary. SBP and DBP in ODM and controls were compared. Subgroup analysis was performed according to type of maternal diabetes and offspring gender. A fixed effect meta-analysis was performed, and a random effects analysis where significant heterogeneity was present. Meta-regression was used to test the relationship between offspring SBP and maternal pre-pregnancy BMI.

Results Fifteen studies were included in the systematic review and thirteen in the meta-analysis. SBP was $1.88 \mathrm{mmHg}$ higher in ODM (95\% CI 0.47, 3.28; $p=0.009$ ). The increase in SBP was similar in both offspring of mothers with gestational diabetes $(1.39 \mathrm{mmHg}$ $[0.00,2.77] ; \mathrm{p}=0.05)$ and type 1 diabetes $(1.64 \mathrm{mmHg}[0.09,3.18]$; $\mathrm{p}=0.04)$. Male ODM had higher SBP $(2.01 \mathrm{mmHg}[0.93,3.10]$ $\mathrm{p}=0.0003)$ and DBP $(1.12 \mathrm{mmHg}[0.36,1.88] ; \mathrm{p}=0.004)$ than controls, but the differences in SBP and DBP between female ODM and controls were not statistically significant. Offspring SBP was positively correlated with maternal pre-pregnancy BMI; however, the association was not significant $(\mathrm{p}=0.37)$.

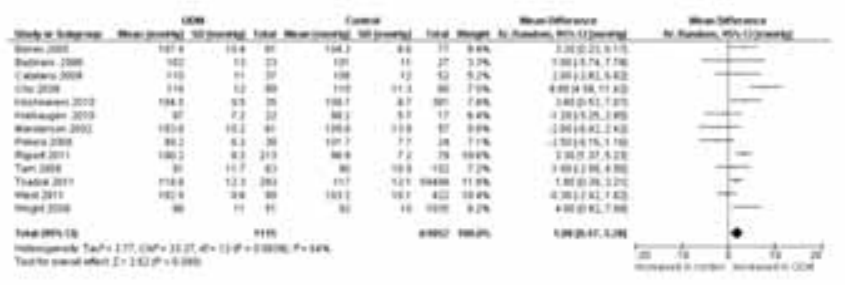

Abstract 1203 Figure 1

Conclusions ODM have higher SBP than controls. This increase is independent of type of maternal diabetes and may be related to maternal pre-pregnancy BMI. Gender-specific differences require further investigation.

\section{UTILITY OF SERUM PROCALCITONIN TO PREDICT RENAL SCARS IN INFANT WITH FEBRILE URINARY TRACT INFECTION}

doi:10.1136/archdischild-2012-302724.1204

B Orive, C Salado, J Len, J Montiano, S Sanchez. Pediatrics, Hospital Universitario de Álava, Vitoria, Spain

Background and Aims The aim of this study was to evaluate the usefulness of Procalcitonin(PCT) as a marker for renal scars in infants with a first febrile urinary tract infection(UTI).

DMSA scintigraphy, the gold standard for detection of cortical scarring, has clearly shown that not all febrile UTI are associated with renal lesions and that common clinical and laboratory evaluations are not reliable to distinguish between acute pyelonephritis(APN) and simple UTI. Scarring secondary to APN is a common event occurring in approximately $30 \%$ of all cases.

PCT, prohormone of calcitonin has been measured in various systemic inflammatory response syndromes, because it appears to be correlated with the severity of microbial invasion and it can be used to check for the presence of parenquimal scars. 
Methods This prospective study includes 140 children less than 2 years, $64 \%$ females admitted for febrile UTI. PCT levels were measured at diagnosis and DMSA scan at the 6 months. We use a study of diagnostic test assessing their sensitivity and specificity. ROC curve was performed.

Results Abnormal DMSA was found in 14 infants (11.3\%). Using a cutoff value of $0.6 \mathrm{ng} / \mathrm{ml}$. PCT sensitivity for detect renal scars on DMSA was $57.1 \%$ (95\%CI, 31.2-83.1\%) and specificity was $56.1 \%$ (95\%CI, 46.7-65.5\%). Negative predictive value was $90.9 \%$ (95\%CI, $84-97.8 \%$ ). AUC (area under curve) is 0.582 .

Conclusions We can conclude that PCT yelds a high negative predictive value of renal damage. Therefore a low PCT value at the time of admission points out a low risk of renal scarring.

\section{INCIDENCE OF ACUTE POST STREPTOCOCCAL GLOMERULONEPHRITIS IN CHILDREN}

doi:10.1136/archdischild-2012-302724.1205

'MM Begolli, ${ }^{2} \mathrm{M}$ Begolli. 'Nephrology, Pediatric Clinic, University Clinical Centre of Kosovo; ${ }^{2}$ Social Medicine, Medical Statistic and Informatic, University of Prishtina, Prishtina, Kosovo

Aim To present a number of children with set diagnosis of APSGN in the Pristina Children's Clinic, incidence, clinical characteristics of the disease an treatment. Their follow up aimed to determine the further course of the disease and the possibility for progressing into a chronic form.

Materials and Methods This was an analysis of the children treated in the Prishtina Children's Clinic during 2009-2011. General date has been taken from all hospitalized patients, including: age, gender, parent's profession, residence and dwelling condition. The diagnosis of APSGN was set in 98 children based an anamnestic date for existing a prior streptococcal infection, characteristic clinical picture, as well as laboratory analyses (hematuria, proteinuria, titer of ASO, determination of the serum creatinin and urea and concentration of C3 and C4).

Results From 98 patients, witch is the total number of the examinees, the majority belonged to the 7-11 year old group, will the male patients were more frequent. According to the obtain anamnestic date, $82 \%$ cases had and anticipatory throat infection. In all cases, the clinical symptoms were hematuria and/or proteinuria. Hypertension was present in $73 \%$ cases, while edema was confirmed in $79 \%$ of the hospitalized patients. Positive ASO titer was confirmed in $75,8 \%$ cases.

Conclusion In a larger number of our patients, i.e. in $96,5 \%$ a complete recovery was obtained with further ambulance follow up, and only $3,5 \%$ entered into the form of chronic glomerulonephritis.

\section{ASSOCIATION BETWEEN DIFFERENT ACUTE KIDNEY INJURY (AKI) DEFINITIONS AND MORTALITY IN VERY LOW BIRTH WEIGHT (VLBW) INFANTS}

doi:10.1136/archdischild-2012-302724.1206

'MT Sinelli, 'G Paterlini, ${ }^{2} \mathrm{P}$ Grillo, 'P Tagliabue. 'Fondazione MBBM-Ospedale S.Gerardo, Monza. ${ }^{2}$ Fondazione IRCCS Ca' Granda - Ospedale Maggiore Policlinico, Milan, Italy

Background AKI has been associated with increased mortality rate among VLBW infants. The best creatinine-based definition for AKI is still unclear. Our aim was to correlate mortality and different AKI-definitions.

Methods From January 1st 2005 to December 31st 2011 all VLBW infants born at S. Gerardo Hospital were recruited whenever $\geq 2$ consecutive p-creatinine determined within 48 hours between 3 and 15 days of life were available.

AKI was defined as single creatinine $\geq 1.5 \mathrm{mg} / \mathrm{dl}$ (AKI-1), increase of $\geq 0.3 \mathrm{mg} / \mathrm{dl}$ within $48 \mathrm{~h}$ (AKI-2) or increase of $\geq 50 \%$ within $48 \mathrm{~h}$ (AKI-3).
The statistical concordance between the definitions was evaluated using the Cohen's Kappa coefficient and their association with mortality using uni- and multivariable logistic regression. AKIdefinitions were adjusted for each other and for GA, BW and Apgar score.

Results Among 263 VLBW infants, 28 (10.6\%), 40 (15.2\%) and 26 (9.9\%) met the definition for AKI-1, AKI-2 and AKI-3 respectively. Low agreement was shown between AKI-1/AKI-2 (Kappa 0.43, 95\%CI:0.27-0.59) and AKI-1/AKI-3 (Kappa 0.32, 95\%CI:0.14-0.51). Substantial agreement was observed for AKI-2/AKI-3 (Kappa 0.69, 95\%CI:0.56-0.82).

$68 / 263$ patients died (28.8\%), with AKI-1 45.1\%, AKI-2 32.5\% and AKI-3 $26.9 \%$ respectively.

AKI patients run higher risk of death than the others (Crude OR $13.6[\mathrm{P}<0.001], 6.7[\mathrm{P}<0.001]$ and 3.8 [P0.007] for AKI-1, AKI-2 and AKI-3).

Using multivariable model, AKI-1 and AKI-2 remained associated with higher mortality (OR $4.25[\mathrm{P}=0.008]$ and $\mathrm{OR} 3.70$ $[\mathrm{P}=0.041]$ ).

Conclusions Different AKI-definitions lead to substantially different patients classifications. Even minimal increment of creatinine are associated with augmented risk of death among VLBW infants.

\section{TREATMENT AND DIAGNOSIS OF THE NEPHRITIC SYNDROME IN CHILDREN}

doi:10.1136/archdischild-2012-302724.1207

${ }^{1} \mathrm{AY}$ Naushabayeva, ${ }^{2} \mathrm{BA}$ Abeuova, ${ }^{1} \mathrm{G}$ Chingayeva, ${ }^{3} \mathrm{~A}$ Nurbekova, ${ }^{1} \mathrm{~K}$ Kabulbayev, ${ }^{2}$ AB Kuzgibekova. 'Nephrology, The Kazakh National Medical University, Almaty; ${ }^{2}$ Pediatrics and Neonatology, Karaganda State Medical University, Karaganda; ${ }^{3}$ Endocrinology, The Kazakh National Medical University, Almaty, Kazakhstan

Background and Aims Nephritic syndrome (NiS) is of significant concern in Pediatric Nephrology with high progression rate. Aim of our study was to establish the pathohistological pattern, and assessment of mofetil mycophenolate efficacy (MMP) in comparison with cyclophosphamide (CYC) in children with NiS.

Methods Study was conducted in 27 children (16 boys) with chronic NiS. Kidney biopsy was performed in all patients under US-guidance using biopsy gun. Pathohistological investigation of renal biopsy included: light, immunofuorescent and electron microsopy.

Results Most frequent pathohistological variant was IgAnephropathy (IgA-NP) $(74.1 \%, \mathrm{p}<0.001)$. In $14.8 \%$ patients NiS was associated with hereditary nephritis. Membranoproliferative glomerulonephritis (GN) (3.7\%), and extracapillary GN (3.7\%) were observed rarely. To induce the remission we used IV methylprednisolone for 3-6 days, oral prednisolone (Pred) $60 \mathrm{mg} / \mathrm{m}^{2} /$ day, MMP $1 \mathrm{~g} / \mathrm{m}^{2} /$ day for 3-4 months. Remission was established when proteinuria was decreased to $0.5 \mathrm{~g} /$ day. Maintenance therapy was administered for one year or longer. Controls were administered with IV (3-4 pulses) or oral (for 2 months) CYC, Pred $60 \mathrm{mg} / \mathrm{m}^{2} /$ day for $1.5-2$ months with following alternating schedule. All patients have received ACE inhibitors. Proteinuria was significantly $(p<0.05)$ lower in main group $(0.1 \mathrm{~g} /$ day $)$ in comparison with controls $(0.9 \mathrm{~g} /$ day), and GFR increasing was more prominent in main group (from 64.3 to $98.7 \mathrm{ml} / \mathrm{min} / 1.73 \mathrm{~m}^{2}$ ), than in controls (from 68.5 to $\left.89.1 \mathrm{ml} / \mathrm{min} / 1.73 \mathrm{~m}^{2}\right)(\mathrm{p}<0.05)$.

Conclusions Thus, chronic nephritic syndrome in children was mostly associated with IgA-nephropathy. Combination treatment with mycophenolate mofetil + steroids and ACE inhibitors is more effective and safe than cyclophosphamide treatment.

\section{NEW BIOMARKERS IN SCREENING AND DIAGNOSIS OF VESICOURETERAL REFLUX}

doi:10.1136/archdischild-2012-302724.1208 